\title{
Cross-Media Color Matching Using Neural Networks
}

\author{
E. Boldrin, R. Schettini \\ Institute of Multimedia Information Technologies (ITIM) \\ National Research Council (CNR) \\ Via Ampere 56, 20131, Milano, Italy \\ E-mail: Centaura@ITIM.MI.CNR.IT
}

\begin{abstract}
Cross-media color reproduction is receiving a great deal of attention as a result of the increasing availability of color devices. A practical approach to accurate color reproduction, integrating colorimetric and interactive methods by means of feedforward neural networks trained by back-propagation, is proposed. Experimental results confirming the feasibility of this approach are reported.
\end{abstract}

\section{Introduction}

Cross-media color reproduction demands device- and viewing condition- independent color description. While most color management systems already incorporate device profiles that make colorimetric, i.e. device independent, color reproduction possible, they often neglect color appearance modeling. At the moment there is not even any general agreement as to the choice and use of a color appearance model, which also requires expensive measuring devices, tightly controlled viewing conditions, and rigorous device modeling and calibration [1]. Thus, the most effective and common approach to the matching of colors on different devices or supports is to have the user define the color combinations interactively. Taking this state of affairs as our point of departure, we propose a practical method, that integrates colorimetric and interactive methods by means of neural networks, for the accurate reproduction of surface color ranges and images on displays. Experimental results are encouraging.

\section{Cross-media color matching by back-propagation}

An early had verified the feasibility of using a feed-forward neural network trained by back-propagation on a small, predefined set of colors to support the entry of color data bases in the context of textile CAD [15]. Color ranges were reproduced colorimetrically on the display, and the user was given editing tools with which to match the colors of the training set. The neural network learned the correct response, using the device-independent color descriptions of the mapping interactively defined by a user. The implicit mapping coded in the neural network was then applied to correct all the colors of the color range to be reproduced on the screen. Although the 
users considered the matches obtained interactively good, the small, predefined, training set (less than 50 elements) was not sufficient to train the network. It was able to learn the training set correctly, but the corrections proposed for the other samples, while congruent with those of the user, were still not enough to dispense with further editing. Larger and more complete training sets appeared to be needed. The procedure presented here has been designed to deal with this drawback, minimizing user efforts.

\section{Color ranges are measured using a spectrophotometer or a colorimeter.}

2. Color ranges are reproduced colorimetrically on the display.

This involves a colorimetric characterization of the device that defines an appropriate mapping between the device-dependent RGB and standard CIE tristimulus values [15]. Since original and reproduced colors are normally seen in quite different contexts and lighting conditions, a chromatic adaptation model is applied. We have chosen here the simplest and most widely used: the CIELAB color appearance model [19]. The following procedure can, however, be applied to correct colors reproduced using any appearance model for a first rendering on the display (the Hunt color appearance model can be used to improve the match $[3,6])$. Unreproducible colors are approximated by projecting them onto the surface of the monitor gamut.

3. The user is given editing tools based on visual interaction with which to match the colors of a suitable training set on the display [14].

\section{A neural network learns to correct the colors to be displayed using the CIELAB device-independent color description of the mapping interactively defined by the user.}

A multilayer feed-forward neural network, consisting of three input and three output units and three hidden layers of 7 units each is used. This architecture was determined empirically [3]. The first, or input layer serves as a holding site for the values to be processed by the network (CIELAB coordinates of the color to be corrected). The last, or output layer is the point at which the final state of the network can be read (CIELAB coordinates of the matching color). Links connect each unit of one layer only to units in the next layer. The activation function of the nodes of the input layer is linear, while that of the hidden layers is sigmoidal. The back-propagation algorithm gives the prescription for changing the weights of any feed-forward network so it can learn the training set constituted by a set of input-output data. This algorithm is designed to reduce the error between the current and the desired output of the network, using gradient descent. A detailed derivation of the back-propagation algorithm can be found in references $[4,12]$. The learning rate and momentum constant are adaptively modified during the iterative learning process, while the training set is randomized to produce an unbiased mapping. The training is continued for as many epochs as are necessary to reduce the overall error of the training set to an acceptably low value. The network is initialized by training it on a set of 24 color samples that cover a wide range of chromaticity.

5. The implicit mapping coded in the neural network is applied to correct subsequent colors of the color range to be reproduced on the screen. 
6. The user is allowed to modify the colors proposed and run the learning phase again.

The system memorizes the mappings corrected by the user during data entry; when a significant number of new mappings have been defined, the user reruns the learning phase. This last step can be repeated during data entry until no further corrections are necessary.

\section{Cross-media color image matching}

Some processing steps must be modified to extend the procedure described above to pictorial images in cross-media matching. This procedure is graphically depicted in Figure 1. When dealing with images, colors cannot be acquired by a spectrophotometer or colorimeter (Step 1), but must be acquired by scanning. Each device has its own color space, defined by the relationship between the input colors and the corresponding RGB codes used to represent them [11]. Consequently, waiving device calibration, which converts the native color space into a standard device-independent one, will result in unmatched colors throughout the system. Moreover, images acquired with different devices can not be reliably compared and stored. Several authors have shown that a $3 \times 3$ matrix transformation can be used to relate $\mathrm{RGB}$ values to $\mathrm{CIE}$ tristimulus values $\mathrm{XYZ}$ if and only if the digitizer channel sensitivities are a linear transformation of the color matching function defining the CIE colorimetric observer e.g. [5]. Relatively few of the scanners and cameras that can be found on the market at present have been designed to meet this constraint, motivating several different approaches to the problem. Strachan et al. [18] describe a method to determine the linear transformation that "best" relates RGB and the CIE $X Y Z$ values. While effective, this method may generate unreliable results when the actual filter responses are far from being a linear transform of the human cone responses. To overcome this drawback Kang and Anderson [8,9] have experimented both multiple linear regression and cascade correlation neural networks for scanner calibration. Although the latter have produced good results on the training sets adopted, the generalization results obtained are rather poor. We have applied a feedforward neural network trained by back-propagation to relate the output of our noncolorimetric scanner (a Sharp JP600) with CIELAB standard coordinates [16]. The training set the algorithm uses is the ANSI IT8 7.2 color target, designed specifically for scanner calibration [10]. Any set of colors not in the training set may be used to test the network's capability of generalization numerically. The Macbeth ColorChecker [16] was chosen for out experimental application so, that the method we propose could be compared with others that have used the same chart to evaluate calibration accuracy. Despite its simplicity, the calibration accuracy of the method is superior to that reported by other authors in similar experiments $[8,9,18]$. A second important difference is the color editing tool (Step 4). Since we can not modify the image appearance by editing single colors, and global modification of the image's appearance often does not result in correct matching, a color cluster editor is needed. We have applied the original Kanamori and Kotera idea of selective color editing [7] 
to develop a soft color cluster editor which allows the user to correct or modify the image colors as they appear on the display, in a friendly and effective way, until satisfactory visual matching with the original is obtained [17].

A color cluster is composed of colors that are "similar" to a selected color centroid; the farther a color lies from the centroid, the less it will be changed in editing. In order to effectively define the cluster we have exploited sighting, the best medium for color communication, and the fact that computer-driven displays allow the user to select and view colors forming composite images on the screen in real time. Visual interaction allows the user to select color centroids without considering their internal representation, physical qualities, or names. When only small precise color adjustments are required, this can be done by clicking on the color of the image (zoom facility is provided), or browsing the image look-up table, or using a color editor [14]. Our experience, however, suggests that taking the color centroid averaging the colors of an image region interactively selected by the user is more suitable for broader color adjustments. Consequently this option has also been made available. As said above, a cluster is composed of colors "similar" to the centroid. Similarity is a typically fuzzy concept. In previous studies we defined three primary fuzzy sets corresponding to similarity in the lightness $\left(\mathrm{L}^{*}\right)$, hue $\left(\mathrm{h}^{*}\right)$ and chroma $\left(\mathrm{C}^{*}\right)$ color dimensions, and used them to permit color image retrieval by pictorial example [2]. These fuzzy sets are used here as the default in the cluster editor.

Since for larger color adjustments the user generally chooses a region of the image to define the cluster, color feature membership functions are defined using the statistical data of the region selected. More precisely, for both lightness and hue a trapezoidal membership function is defined, with a major bases four times the feature's standard deviation and a minor bases twice the standard deviation. For chroma the major bases is set at six times its standard deviation (these numbers were defined in preliminary elicitation experiments).

The membership functions defined, $\mu_{\mathrm{h}^{*}}\left(\Delta \mathrm{h}^{*}\right), \mu_{\mathrm{c}^{*}}\left(\Delta \mathrm{C}^{*}\right)$, and $\mu_{\mathrm{L}^{*}}(\Delta \mathrm{L} *)$, associate a degree of similarity of a given color feature (hue, chroma, or lightness) with respect to the same feature of the color centroid. The product of these three membership functions is used to define the cluster membership function [17], i.e. the degree of similarity of a given color to the cluster centroid:

$$
\mu_{\mathrm{h}^{*} \mathrm{C}^{*} \mathrm{~L}^{*}}\left(\Delta \mathrm{h}^{*}, \Delta \mathrm{C}^{*}, \Delta \mathrm{L}^{*}\right)=\mu_{\mathrm{h}^{*}}\left(\Delta \mathrm{h}^{*}\right) \mu_{\mathrm{C}^{*}}\left(\Delta \mathrm{C}^{*}\right) \mu_{\mathrm{L}^{*}}\left(\Delta \mathrm{L}^{*}\right)
$$

When $\mu_{\mathrm{h}^{*} \mathrm{C}^{*} \mathrm{~L}^{*}}\left(\Delta \mathrm{h}^{*}, \Delta \mathrm{C}^{*}, \Delta \mathrm{L}^{*}\right)=1$, that is $\mu_{\mathrm{h}^{*}}(\Delta \mathrm{h} *)=\mu_{\mathrm{L}^{*}}\left(\Delta \mathrm{L}^{*}\right)=\mu_{\mathrm{C}^{*}}(\Delta \mathrm{C} *)=1$, the color considered will be modified in the described manner. When $\mu_{\mathrm{h}^{*} \mathrm{C}^{*} \mathrm{~L}^{*}}\left(\Delta \mathrm{h}^{*}, \Delta \mathrm{C}^{*}, \Delta \mathrm{L}^{*}\right)=0$, that is, when at least one of the membership functions is zero, the color will not be changed in editing, because it does not belong to the cluster. When $0<\mu_{\mathrm{h}^{*} \mathrm{C}^{*} \mathrm{~L}^{*}}\left(\Delta \mathrm{h}^{*}, \Delta \mathrm{C}^{*}, \Delta \mathrm{L}^{*}\right)<1$, the color will be modified in proportion to its similarity to the centroid.

Since similarity is a subjective, context-sensitive property, there are several situations in which default, or statistically defined membership functions do not coincide with the user's idea of "similarity". Rather than model all the possible situations, we have again taken advantage of the possibility of having a visual feed-back of the user's 
choices: the system displays a gray-level image $B(x, y)$ in which the darkness of a pixel corresponds to its degree of similarity to the centroid considered. This picture is easily obtained as follows:

$$
\mathrm{B}(\mathrm{x}, \mathrm{y})=\left[\left(\mathrm{L}^{*}{ }_{\mathrm{bg}}-\mathrm{L}^{*}{ }_{0}\right)\left(1-\mu_{\mathrm{h}^{*} \mathrm{C}^{*} \mathrm{~L}^{*}}\left(\Delta \mathrm{h}^{*}, \Delta \mathrm{C}^{*}, \Delta \mathrm{L}^{*}\right)\right)\right](\mathrm{x}, \mathrm{y})
$$

where $\mathrm{L}^{*}{ }_{\mathrm{bg}}$, and $\mathrm{L}_{0}{ }_{0}$ are the background and black-point lightness respectively.

Figure 2 illustrates color centroid selection using the color editor we have developed. The default membership functions are given in the lower window of the Figure, while the gray-level picture on the right shows the pixels that would be modified in editing (the darker they are, the more similar they are to the centroid). The user can move the sliders provided to define the shape of the feature membership functions and have an immediate visual feed-back of the derived cluster membership function (the feature membership functions thus defined may have a rectangular shape: this corresponds to a crisp definition of the cluster boundaries).

The appearance of the cluster defined can be modified by changing one color feature at a time. Altogether the user can modify seven interindependent features: hue $\left(h^{*}\right)$, lightness $\left(L^{*}\right)$, chroma $\left(C^{*}\right)$, redness $\left(r^{*}\right)$, greenness $\left(g^{*}\right)$, yellowness $\left(y^{*}\right)$, and blueness $\left(b^{*}\right)$ (the last four variables correspond to positive and negative variations of the $u^{*}$ and $v^{*}$ features). Given any possible color changes selected by the user, the new color coordinates of the cluster colors are modified proportionally to their degree of similarity to the cluster centroid.

The user has an immediate visual feed-back of the editing activity. If a part of a cluster is moved outside the display gamut during editing, the system gives an acoustic warning and automatically performs an approximation of the out-of-gamut colors by desaturating them. Once the cluster has been modified to the user's satisfaction, the whole procedure can be repeated for different colors. Colors already edited can be protected so that they will not be inadvertently altered in successive operations.

The same network topology and learning strategy used in the first experiment have been adopted for the color appearance mapping of the image to be reproduced (the learning rate and momentum constant are adaptively modified during the iterative learning process, while the training set is randomized to produce an unbiased mapping). To initialize the network a picture of the IT8 7.2 is reproduced on the screen and the user is allowed to modify its colors with the soft color cluster editor until he feels it matches the appearance of the original picture. The initial training set is composed of all the colors thus modified.

\section{Preliminary results}

The method proposed here has been proved effective when tested with the assistance of a team of three users familiar with color manipulation, on real data within the framework of textile CAD. The data entry of color ranges in a CAD system is a sequential process which is usually performed by a trained user through a trial and 


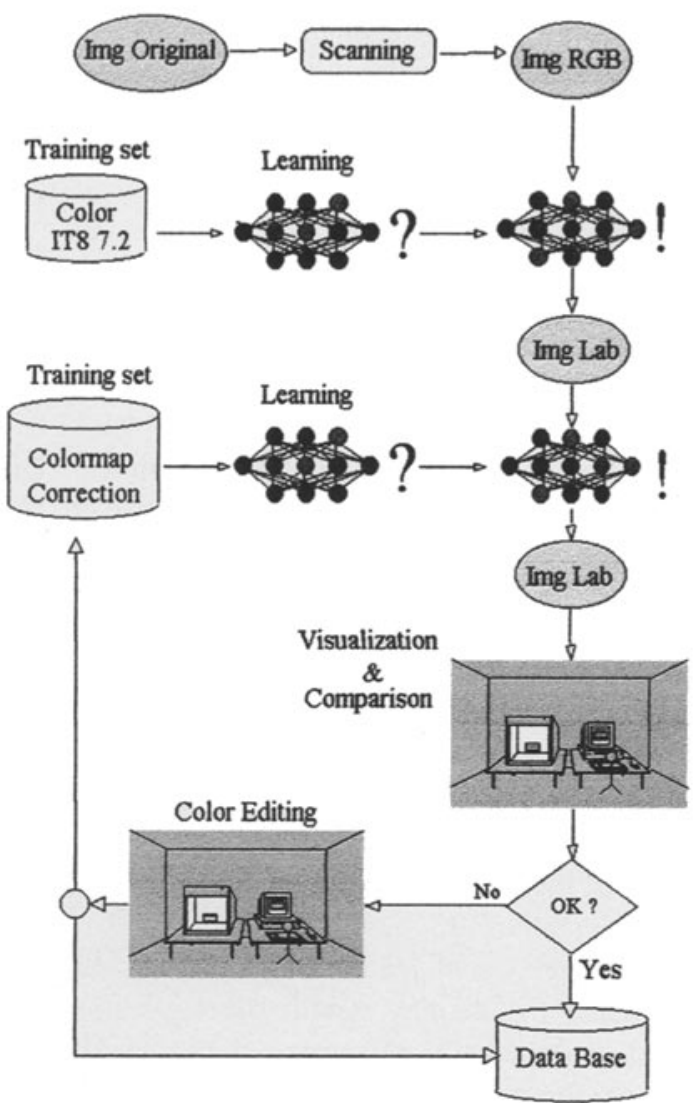

Fig. 1: The strategy developed strategy for cross-media color image matching.

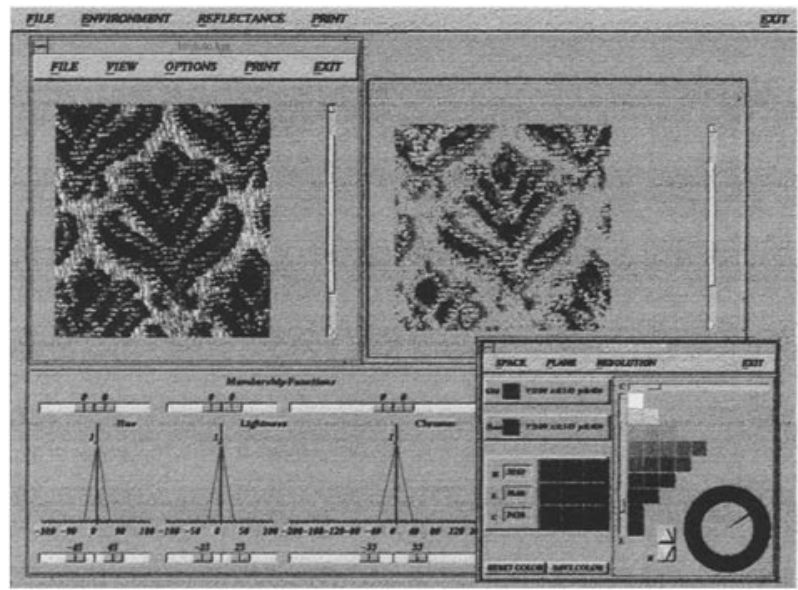

Fig.2: Color centroid selection using the color editor. The similarity picture and default membership functions are also shown 
error approach. Using our approach the entry of a color range of over 800 samples in silk was begun by applying the color correction proposed by the network trained on a set of 24 color samples randomly chosen. Each user then "personalized" the training set, modifying some of the color mappings proposed by the network during data entry. Subsequent colors were entered at random (although within the CAD system the users could organize and browse them in several different ways), selecting only colors that were likely to be used together with those colors already reproduced. When at least 25 mappings had been redefined during color entry, the user ran the learning phase again.

We found that the mapping proposed by the neural network after the first training stage reduced the average correction of new colors by about half for all three users. Further personalization of the training set produced less regular results for different users; however, with a training set of about 100 elements the network learned to model each user well enough to require only user supervision in subsequent color data entry.

The procedure has been also been extended successfully to pictorial cross-media image in trial experiments regarding the entry of more than 200 images in a multimedia catalogue of ancient textiles. The mapping proposed by the neural network after the first training stage always improved the quality of the image matching. Subsequent color corrections generally reduced the average color distance between the CIELAB coordinates of the image colors proposed by the network and those of the matching colors obtained by the users' editing. Sometimes, however, the color harmony of the image was modified unexpectedly, making further interactive corrections necessary. The method has still to be tested on different image databases and its effectiveness evaluated in paired-comparison with more standard color correction techniques [1]. We must also solve a "technical problem" related to the color correction network design. During the acquisition of the image database many thousands of colors are "more or less" modified. If the colors in the training set are over four times the number of network links, the network may no longer be able to generalize the desired mapping. To overcome this drawback a maximum must be set for the size of the training set, or else the network must be allowed to evolve dynamically as the training set grows [4]. In the present version we add to the original training set (the color corrections of the IT8 7.2 color set) only centroids of the color clusters that have been modified. We are, however, exploring other hypotheses.

Several experiments are currently in progress to increase the effectiveness of application of our procedure [3] and evaluate them according to CIE guidelines [1].

\section{References}

1. P.J. Alessi "CIE guidelines for coordinate research on evaluation of colour appearance models for reflection print and self-luminous display image comparison" Color Research and Application Vol. 19, pp. 48-58, 1994. 
2. E Binaghi, I Gagliardi, R. Schettini "Image retrieval using fuzzy evaluation of color similarity" International Journal of Pattern Recognition and Artificial Intelligence, Vol. 8, pp. 945-968, 1994.

3. E. Boldrin, P. Campadelli, R. Schettini "Effective and efficient mapping of color appearance" Color Research and Application, 1997 (in print).

4 J. Hertz, A. Krogh, R.G. Palmer "Introduction to the theory of neural computing" Addison-Wesley, New York, Vol. 1, pp. 115-162, 1991.

5 B.K.P. Horn (1984) Exact Reproduction of Colored Images, Computer Vision, Graphics and Image Processing, Vol. 26, pp. 135-167.

6 R.G.W. Hunt "Revised colour-appearance model for related and unrelated colours" Color Research and Application, Vol. 16, pp. 146-165, 1991.

7. K. Kanamori, H. Kotera "A Method for Selective Color Control In Perceptual Color Space" Journal of Imaging Technologies, Vol. 35(5), pp. 307-316, 1991.

8 H.R. Kang P.G. Anderson "Neural network application to the color scanner and printer calibrations" Journal of Electronic Imaging, Vol. 1, pp. 25-135, 1992.

9 H. Kang "Color Scanner Calibration. Journal of Imaging and technology", Vol. 36, 162-170, 1992.

10 N. Otha "Development of color targets for input scanner calibration" Proc. 7th Congress of the International Color Association "Colour 93", Technical University of Budapest, Budapest (Hungary), p. 130, 1993.

11 Proc. 3nd IS\&T and SID's Second Color Imaging Conference: Color Science, Systems and Applications, 1995.

12 D.E. Rumelhart, G.E. Hinton, R.J. Williams "Learning internal representations by error propagation" In D.E. Rumelhart, J.L. McClelland Eds, Parallel distributed processing,, MIT Press, Cambridge (MA), Vol. 1 pp. 145-168, 1986.

13. H. Saarelma, P. Oittinen "Automatic Picture Reproduction" Graphics Art in Finland, Vol. 22(1), pp. 3-11, 1993.

14. R. Schettini, A. Della Ventura, M.T. Artese "Color Specification by Visual Interaction" The Visual Computer, Vol 9(6), pp. 143-150, 1992.

15 R. Schettini "The faithful rendition of color ranges on display, Proc. IS\&T and SID's Color Imaging Conference: Transforms \& Transportability of Color, Scottsdale, Arziona, pp. 160-163, 1993.

16 R. Schettini, B. Barolo, E. Boldrin "Colorimetric calibration of color scanners by back propagation" Pattern Recognition Letters, Vol. 16(10), pp. 1051-1056, 1995.

17 R. Schettini, B. Barolo, E. Boldrin "A soft color cluster editor" Image Processing and Communications, Vol. 1(1), pp. 17-32, 1995.

18 N.J.C. Strachan, P. Nesvadba e A.R.Allen "Calibration of video camera digitising system in the $\mathrm{L}^{*} \mathrm{u}^{*} \mathrm{v}^{*}$ colour space" Pattern Recognition Letters, 11, 771-777, 1990.

19 G. Wyszecki e W.S. Stiles "Color science: concepts and methods, quantitative data and formulae", Wiley, New York, 1982. 